\title{
Detecting Resonances using Evolutionary Algorithms
}

\author{
Y.G. Petalas ${ }^{1}$, C.G. Antonopoulos ${ }^{2}$, T.C. Bountis ${ }^{2}$ and M.N. Vrahatis ${ }^{1,3}$ \\ ${ }^{1}$ Computational Intelligence Laboratory (CI Lab), Department of Mathematics, \\ University of Patras Artificial Intelligence Center (UPAIRC), \\ University of Patras, GR-26110 Patras, Greece \\ ${ }^{2}$ Center for Research and Applications of Nonlinear Systems (CRANS), \\ Department of Mathematics, University of Patras, Greece
}

\begin{abstract}
We propose a new approach for the identification of the resonances appearing in symplectic maps. In the proposed methodology, we make use of Evolutionary Algorithms which are population based search strategies used for global optimization. We have applied the proposed methodology to the 2 - dimensional (2D) Hénon map and obtained promising results which can be generalized to symplectic maps of higher $(2 m)$ dimensions. As is wellknown, such maps are representative of Hamiltonian systems and occur in many physical applications.
\end{abstract}

Keywords: discrete dynamical systems, maps, resonances, evolutionary algorithms

Mathematics Subject Classification: 37J10, 90C15

PACS: 29.20.Fj, 29.27.-a, 29.27.Bd

\section{Introduction}

In this paper, we propose a new methodology for the numerical detection of resonances in symplectic maps of even dimension. We formulate this task as an optimization problem. Evolutionary Algorithms [1] (EAs) are then used to solve this problem.

Evolutionary Algorithms [1] (EAs) are population based heuristic optimization algorithms, which use mechanisms inspired from biological evolution, such as crossover, mutation and natural selection. EAs attain a set of possible solutions, called "population", using an objective function to evaluate each candidate solution also named "an individual". Evolution of the population then takes place after the repeated application of the above mechanisms (also called operators).

Examples of EAs are the genetic algorithms (GAs) [2], the evolution strategies [3] and the genetic programming [4] and Differential Evolution Algorithms (DE) [5]. Related to EAs is the Particle Swarm Optimization (PSO). PSO belongs to the class of Swarm Intelligence algorithms, which are inspired from (and based on) social dynamics and emergent behavior in socially organized colonies [6]. In the context of PSO, the population is called a swarm and the individuals (or search agents) are called particles. Both, DE and PSO have proved very efficient and easy to use in a great variety of optimization problems.

The above methods are particularly effective when the objective function is not differentiable and/or is discontinuous or the objective function is multimodal. They are also very simple in

\footnotetext{
${ }^{3}$ Corresponding author: e-mail: vrahatis@math.upatras.gr, Phone: +30 2610 997374, Fax: +30 2610992965
} 
their use and implementation, while they require only function evaluations without needing the computation of function's derivatives.

Finally, very promising and effective are the Memetic Algorithms. Memetic Algorithms (MAs) are metaheuristic-search algorithms used to solve global optimization tasks. MAs are hybrid algorithms which combine EAs and local search methods. EAs achieve exploration of the whole search space and local search methods exploit smaller regions [7].

\section{The Proposed Approach, Results and Discussion}

The goal of the proposed methodology is the numerical detection of resonances (or sequences of stable and unstable periodic orbits) of maps of a $2 m$-dimensional space onto itself. In the 2-dimensional case, the method works as follows: We choose a series of concentric circles with increasing radii around the main elliptic point of the map. In the region contained between two consecutive circles, we initialize a population of 10-20 individuals of an EA. In this framework, the individuals of the EA population are points of the phase space of the map. For every such individual, we take its coordinates and iterate them through the map for $10^{4}$ iterations, measuring the minimum and the maximum distance of the produced set of points from the central elliptic point of the map. The fitness function of the EA is the difference $D$ between the maximum and minimum distance $(D=$ maximum - minimum) from the origin of the map of all these orbits and the goal of the EA is to maximize the quantity $D$. The reason is that $\mathrm{D}$ experiences large variations precisely where there are big "islands" in phase space corresponding to the location of relatively low order resonances (or periodic orbits of small period $N$, where $N$ is of order 10 or 20).

Thus, plotting the associated $D$ values versus the radius of the individual orbits produces a function whose maxima and minima are expected to give us an indication of the existence of resonances at the corresponding radius.

We have applied our method in the 2D Hénon map for the frequency $v_{x}=0.45$. The $2 \mathrm{D}$ Hénon map is given by the set of equations

$$
\begin{aligned}
& x^{\prime}=\cos \left(2 \pi v_{x}\right) x+\sin \left(2 \pi v_{x}\right)\left(p_{x}+x^{2}\right), \\
& p_{x}^{\prime}=-\sin \left(2 \pi v_{x}\right) x+\cos \left(2 \pi v_{x}\right)\left(p_{x}+x^{2}\right) .
\end{aligned}
$$

and is known from its application e.g. to the dynamics of particle beams in high energy accelerators [8]. Its phase space picture is shown here in Figure 1. Note the different chains of islands in this figure associated with resonances of different order: The lower the order (and the smaller the period of the periodic orbits) the bigger the size of the islands. For example, after a region of smoothly varying concentric curves, we notice in Figure 1 (at a distance of about 1.2 from the origin) a dramatic change in the morphology of the orbits caused by the presence of a period 7 resonance. Then the closed curves become smooth again until another dramatic change occurs at about 1.6 units from the origin, where a chain of 12 islands can be clearly seen in the Figure 1.

Let us see all this now in Figure 2, where we present a plot with the corresponding results of our EA strategy. We have used many values for our concentric radii, forming a series of annuli lying in the interval $[0.2,3.0]$, with step 0.01 . For every such annulus, we record the output $D$ found by the EA. Specifically, here, as a first candidate from the class of EAs we have used the Differential Evolution algorithm [5] which is, in general, very effective and efficient.

Notice in Figure 2(a) the 2 local major maxima of $D$ at distances 1.25 and 1.6 approximately, where $D$ experiences sudden upward jumps. These jumps correspond precisely to the two sudden changes in the morphology of the dynamics observed in Figure 1 near the resonances of period 7 and 12. In fact, there is a lot more to this figure than meets the (naked) eye! If we magnify the region between the two big jumps (see Figure 2(b)), we notice a very small one near a radius of about 1.49. This corresponds to a resonance of 19 islands, barely visible also in Figure 1. There are also 


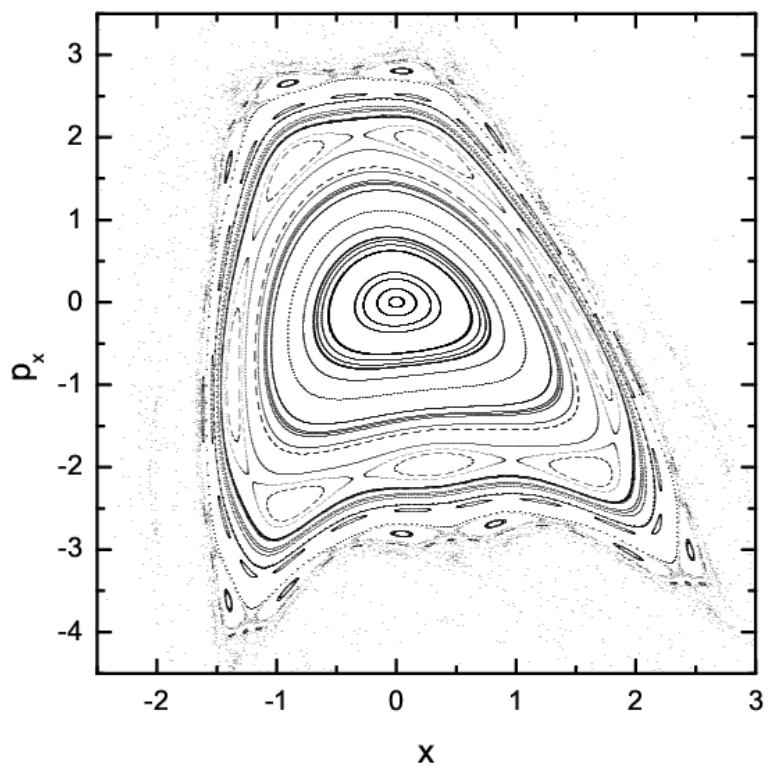

Figure 1: The phase space of the 2D Hénon map for the frequency $v_{x}=0.45$.

other local minima and maxima in Figure 2(a), following the second big jump, which correspond to chains of islands of different periods near the big chaotic regions outside where orbits escape to infinity.

In a future work, we intend to make a comprehensive study of this picture, using a variety of EA (mentioned in Section 1) and try to conclude which one is more efficient in depicting the remarkable morphology of the dynamics, not only for $2 \mathrm{D}$, but also for higher dimensional maps of interest to accelerator dynamics [9].

\section{Acknowledgment}

This work was supported by the "Pythagoras I" research grant co funded by the European Social Fund and National Resources. It was also partially supported by the European Social Fund (ESF), Operational Program for Educational and Vocational Training II (EPEAEK II) and particularly the Programs HERAKLEITOS, providing a Ph.D scholarship for one of us (C.G.A.) and PYTHAGORAS II, supporting in part the research of T.C.B.

\section{References}

[1] Engelbrecht A. P., Computational intelligence: an introduction. John Wiley and Sons, 2002.

[2] Holland J. H., Adaptation in natural and artificial systems. MIT Press, 1975.

[3] Schwefel H. P., Evolution and optimum seeking. Wiley, New York, 1995.

[4] Koza J. R., Genetic programming: on the programming of computers by means of natural selection. MIT Press, Cambridge, MA, 1992. 


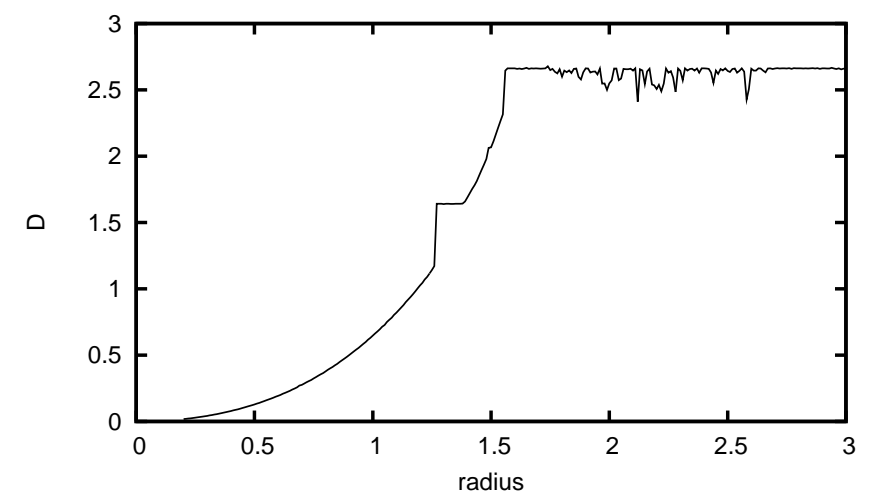

(a) Original plot

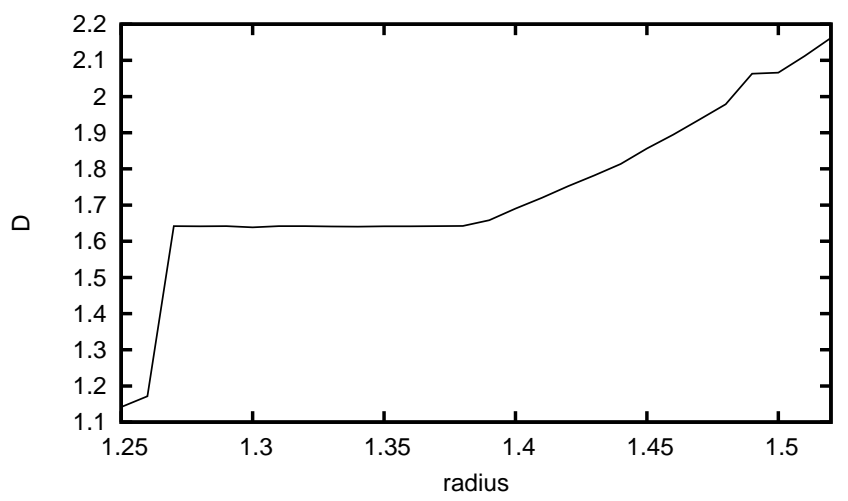

(b) A magnification of Fig. 2(a)

Figure 2: The proposed methodology was applied in the 2D Hénon map. The horizontal axis denotes the radius and the vertical axis the output difference $D$ as a function of the radius. In Fig. 2(a) is the original plot while in Fig. 2(b) a magnification of Fig. 2(a) is depicted.

[5] Storn R. and Price K., Differential evolution - a simple and efficient heuristic for global optimization over continuous spaces. Journal of Global Optimization, 11(4) 341-359(1997).

[6] Kennedy J. and Eberhart R. C., Swarm intelligence. Morgan Kaufmann Publishers, 2001.

[7] Krasnogor N. and Smith J., A tutorial for competent memetic algorithms: model, taxonomy and design issues. IEEE Transansaction on Evolutionary Computation, 9(5) 474-488(2005).

[8] Scandale W. and Turchetti G. (eds.), editors., Nonlinear problems in future particle accelerators. World Scientific, Singapore, 1991.

[9] Vrahatis M. N. Isliker H. and Bountis T. C., Structure and breakdown of invariant tori in 4D mapping model of accelerator dynamics. International Journal of Bifurcation Chaos, 2707-2722(1997). 